\section{Ueber den gegenwärtigen Stand der Erblichkeitsfrage in der Lehre von der Tuberculose.}

\author{
Von \\ Dr. M. Wahl in Essen. \\ (Fortsetzung aus No. .̈.)
}

II.

Die als "erbliche Tuberculose" bezeichnete Krankheit tritt il den einzelnen Geschlechtern in verschiedener Art und Weise zu Tage, lnd ist man bestrebt gewesen, nach den jeweiligell Auffassungen die einzelnen Formen in verschiedene Systeme hineinzupassen. Bollinger ${ }^{1}$ ) zählt die einzelnen Arten der Vererbung auf, und kann man nacl ihm von directer Vererbung sprechen, weln die Krankheit der Elterll von einer oder der anderen Seite auf die Kinder übergeht, sind beide Eltern krank, dann limmt man eine potenzirte oder combinirte Erblichkeit an. Wenl einzelne oder mehrere Generationen übersprungen werden, und die Vererbung von Grosseltern oder Geschwisteru der Eltern ausgeht, dann ist eine indirecte oder laterale vorhanden, Formen, die zum Theil auch als Atavismus bezeichnet werden. Collaterale Erblichkeit ist diejenige, wenn die Geschwister an derselben Krankheit leiden ohne vorherige Erkrankung der Grosseltern und Eltern, gleichartige, wenn die gleiche Form, ungleichartige, weim eine verschiedene Form der Krankheit bei Eltern und Kindern lierrscht. Bollinger führt ferner noch die scheinbare Vererbung, die Pseudoberedität an, wenı unter dem Einflusse übereinstimmender äusserer Verhältnisse wie Beschäftigung, Ernährung, Wohnung etc. sicl bei den Kindern dieselbe Krankheit wie bei den Eltern entwickelt, so dass alsdann öfters der Schein der Vererbung erweckt wird.

Die Pseudohereditat ist nun bei der Tuberculose sehr in Betracht zu ziehen, und fragt es sich zunächst, welche Reihe von Fällen bei der Phthisis unter der Kategorie „Erblichkeit" aufgezählt werden können. - Unter einer wirklichen Vererbung kann in dem angedeuteten Sinne eigentlich nul die parasitäre verstanden werden. Dieselbe ist aber eine fragliche uud höchstels nur ausnahmsweise vorkommende, wenn sie überhaupt vorkommen sollte, und deshalb könnte der Begriff der rerblichen Tuberculose" uur auf ein ganz minimales Gebiet beschränkt bleiben. Andererseits ist nun wiederum die Vererbung einer Krankheitsanlage niclit identisch mit der Erblichkeit der Krallkheit selbst, weil zu jener immer erst wieder Nälırboden und Infection linzutreten müssen, ehe die Krankheit zu Stande kommen kann. Nährboden, Infection und Tuberculose bilden eine geschlossene Kette, aus der beim Zustandekommen der Krankheit kein Glied fehlt. Da nun die durch Heredität überkommene krankhafte Anlage blos die Bedingungen für eine leichtere Herstellung eines günstigeı Nährbodens für eine spätere Infectioll in sich trägt, auf welche die Tuberculose erst folgt, so dürfte die erstere streng genommen auch nur zur Pseudoheredität zu zählen seill. Allein es ist bisher immer Brauch gewesen, unter der Rubrik Tuberculosenerblichkeit auch die , dispositionelle erbliche Belastung", die Vererbung jener im weiteren Sinne als Disposition zu Tuberculose bezeichnete krankhafte Körperbeschaffenheit mit aufzuzählen, und rechnete man bisher im Allgemeinen Schwindsuclitsfälle, wo bei den Eltern, deren Geschwistern oder bei den Grosseltern die nämliche Krankheit vorgekommen, zu den "vererbten". Der Begriff der Tuberculosenerblichkeit bleibt demnach in gewissem Sinne immer ein relativer. Zur Klärung der Verhältnisse hat der eben bezeichnete Unsstand keineswegs beigetragen, jedenfalls muss bei der Beurtheilung der Erblichkeit genau individualisirt werden, und wenn die "dispositionelle" Vererbung mit unter der Kategorie Tuberculosenerblichkeit aufgeführt werden soll, dann müssen alle Fälle von später erworbener Disposition abgetrennt werdell, welche irgendwie geeignet erscheinen, den Eindruck der Heredität zu hinterlassen. Darin liegt aber neben der allatomischen Beweisführung der parasitären Tuberculosenerblichkeit und der Unterscheidung ihrer Formen die Hauptschwierigkeit bei der Lösung der Erblichkeitsfrage ïberhaupt.

」) Bollinger a. a. 0.7.
Die Fälle, welche als ,erbliche" imponiren können, sind recht läufig. Es gehören dahin alle diejenigen, wo aus tuberculösen Familien stammende, aber gesund geborene Individıen sich durch dauernde Anpassung an gewisse ungünstige Lebensbedingulgen und Gewohnheiten, unter deren Einfluss beiläufig auch die Eltern gestanden und welche somit aucl als fortgeerbte betrachtet werdell köunen, eine "Disposition" acquiriren, oder wo die letztere iı Folge voll Gelegenheitsursaclen, wie Krankheiten sich, entwickelı.

Gleiclı ilı den ersten Lebenswoclıen sind eine Reihe von Zuständeıl geeigılet, im Neugeborenen einen günstigen Nährboden für die Bacillen zu scluaffen. Icl nenne nur die Katarrhe des Respirationsund Digestionstractus, voll denen die ersteren so lıäufig durch unrichtige oder mangellafte Pflege und letztere durch Fehler in der Ernählung erzengt werden. Bleiben solcle von Haus aus gesulde Kinder null im Verkehr mit den tuberculösen Eltern oder Ammen, oder erhalten sie ungekochte Milch voll perlsüclitigen Kühen als Nahrung, dann steht einer Infection Nichts im Wege. Solche Fälle gelten aber durchweg als "ererbte", während sie in der That nur Beweise für die Contagiosität der Tuberculose sind, welche häufiger ist als bisher angenommen wurde. -

Der Glaube an die Coutagiosität der Schwindsucht reicht bis ins Alterthum, aus dem Mittelalter liegen gleichfalls Beobachtungen

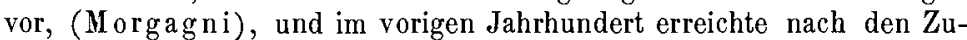
sammenstellungen voll Corradi ${ }^{1}$ ) jener den Höhepunkt. Leichtenstern $^{2}$ ) hat die verschiedenen Ansichten hierüber zusammengestellt und beleuchtet.

Die Stimmell gegen die Ansteckungsfähigkeit der Schwindsucht überhaupt sind vereinzelt und die dagegen angeführten Gründe nicht stichhaltig oder beweisend. Man hat versucht, aus der Krankenhausstatistik namentlich der Londoler Spitäler den Beweis zu führen, dass die Phtliise nicht oder doclı nur sehr wenig ansteckungsfähig sei. Wenn die Statistik des Brompton-Hospitals ${ }^{3}$ ) in 36 Jahrell bei einer hohen Krankenziffer nur 3 wirklich in der Anstalt an Plitliise erkrankte Persollen des Pflegepersonals (1 Arzt, 2 Wärterinnen) aufweist, so ist das doch gewiss nur ein Beweis dafür, dass bei dem dienstthuenden Personal die Bedingungen, welche einer Alısteckung Vorschub leisten, ein günstiger Nährboden, nicht odel nur selten vorlandell waren. Dasselbe gilt von den anderen Zahlenreihen, wie sie in den Berichten ïber Chest Hospital und in dell Erfahrungen von Pollock ${ }^{4}$ ) sowie von Aufrecht über das Magdeburger Hospital enthalten sind. - Andere wie Schnitzler ${ }^{5}$ ) gestehen die Contagiosität wohl zu, meinen aber, sie sei nur auf minimale Grenzen beschränkt. Auch nach Leyden ") spielt die Contagiosität keine grosse Rolle, derselbe glaubt vielmehr der Heredität eill grösseres Terrain zuweisen $\mathrm{zu}$ sollen.

Dagegell haben früher $\mathrm{Baly}^{7}$ ) und lleuerdings Sommerbrodt ${ }^{8}$ ) und $\mathrm{Baer}{ }^{9}$ ) das häufige Auftreten der Tuberculose in geschlossenen Anstalteı, wie lnvalidenhaus und Gefängniss, hervorgehoben und eine Erkläruıg il dem vielfaclıen Zusanmenlebell mit Phthisischen zu fillden geglaubt. - Der berühmte Fall Bergeret von Lichtheim, in den Verhandlıngen des II. Congr. f. inller. Med. erzählt (II, 20), sei hier angefülırt.

Am meisten wird die Ansteckung durch den innigen Verkehr in den Familieı gefördert. Es zeigt dies die tägliche Erfahrung, und bildet namentlich die Uebertragung der Phthise unter Ehegatten den sprechendsten Beweis dafür. Schon die Statistik von H. Weber ${ }^{10}$ ) zeigt dies, derselbe fand bei 80 Ehen, wo der eine Theil mit Phthise behaftet war, in 29 Fällen eine Uebertraguing auf den andern Theil, $18 \mathrm{mal}$ auf die Frau nlld $11 \mathrm{mal}$ auf den Mann. Leudet ${ }^{11}$ ) (Rouen) theilte auf dem Genfer Congresse Beobachtungen aus 28 Jahren in 133 Familien ill mehreren Generationen mit, il 15 Ehen war del Mann phthisisch, davon wurde $5 \mathrm{mal}$ die Frau inficirt, aus 41 Ehen war die Frau bei der Eheschliessung tuberculös, davon wurden in

1) Verhandlungen d. Cienfer internat. hygien. Congr. 1882.

$\Rightarrow$ Leichtenstern, über die Lehre von der Ansteckunsfähigkeit der Schwindsucht im Alterthum, Mittelalter u. d. nevern Zeit" - Gorrespondenzbl. d. ärztl. Vereins für Rheinland, Westfalen 1883. 31.

3) Humphry's Bericht in der Cambridge medical Society, s. I, ancet 1883. 323 .

t) Pollock, Modem thenry and treatment of phthisis, s. Iancet 1883 . S. 718 .

5) Schnitzler, Wiener melic. Presse 1883. No. 49.

6) L eyden, Klinisches iiber i. Tuherkelcacillus. Zeitschr, f. klin. Med. VIlI. 5. 387.

i) Public. Health, Report of the medic. offic. of the Privy Council 1858.

s) Sommerbrodt, Erinnerungen a. d. Berliner Invalidenhause, deutsche militärärztl. Zeitschr. 1883. 4.

9) Baer, Zeitschr. f. klinische Nedicin 1883. 5. 511

10) Sitzungsbericht d. London. Clinical Society 8. Mai 1874.

11) Vierteljahrsschr. f. iffentl. Gesundheitspflege 1882. II. 215. 
3 Fällen der Mann angesteckt. Bockendahl ${ }^{1}$ ) giebt an, dass von 938 Ehelenten, welche an erworbener Schwindsucht zn Grunde gingen, 101 Fälle waren, wo der andere Ehegatte schwindsuichtig wnrde, (10,8 Proc.), bei 729 Ehegatten mit ererbter Phthise war dies $33 \mathrm{mal}$ $(4,5$ Proc.) der Fall, also im Ganzen bei 1667 Fhen 134 Ansteck nngen $(8,1$ Proc.). - Im I. Report of the collect. investigat. board ${ }^{2}$ ) sind 119 Fälle von Ansteckung der Frau durch den Mann und 69 der Männer durch die Frau angegeben. - In meinen Journalen fand ich in 64 Familien mit 197 phthisischen Mitgliedern 20 Ehepaare, wo die Infection $13 \mathrm{mal}$ vom Manne auf die Frau und 7 mal von der Frau auf den Mann überging. Dabei war ein Mann, welcher nach einander in zwei Ehen die Frau inficirte, deren Kinder ebenfalls, wahrscheinlich durch Lactation, tuberculös wurden. - Bezüglich der Ergebnisse der vom Verein f. immere Medicin in Berlin angestellten Sammelforschungen giebt $\mathrm{Ewald}^{3}$ ) an, dass von 1078 Antworten auf die Frage der Uebertragung der Lungentıberculose 261 in bejahendem Sinne eingegangen seien, 158 davon betreffen die Anstecking unter Eheleuten.

Wenn diese Zahlen die Häufigkeit der Anstecknng nnter Ehegatten sattsam beweisen, so lässt sich anch eine mindestens ebenso grosse Uebertragbarkeit zwischen Eltern, namentlich Müttern und Kindern wohl mit Bestimmtheit ammehmen. Wir haben nur keine statistischen Zahlen aufzuweisen. Es dïrfte alber wohl die Vermuthung nicht nngerechtfertigt sein, dass die angedeuteten Contagiositätsfälle, welche bei Kindern Erblichkeit vortänschen, nicht gar zu selten sind.

Es kommen hier drei Importationswege in Betracht, Inhalation, Ernährung und Läsionen der Haut.

Die Versuche Tappeiner:s ${ }^{4}$ ), fortgesetzt von Lippl, Reinstad ler und Berthean ${ }^{5}$ ), liaben den liffect der Inhalation des tulerculösen Giftes experimentell klar gestellt, nachdem man den Hanch der Schwindsüchtigen schon lange vorher fiir gefahrlbringend gehalten hatte. Koch zeigte die Infectiosität der eingetrockneten Sputa, welche mit Bacillen beliaftet in zerstäubtem Znstande in die Luft nnd von $\mathrm{da}$ in die Athmungsorgane gelangen. Seither haben Veraguth ${ }^{6}$ ), Weichselbaum ${ }^{7}$ ) nnd Charles Lajoue ${ }^{8}$ ) durch ihre Versuche die Thatsache der Inhalationstnberculose von Neuem bestätigt. Die zehn von Reich ${ }^{9}$ ) nitgetheilten Fälle, wonach 10 nengeborene Kinder dadurch tuberculös wurden, dass eine phthisische Hebamme denselben von Mund zu Mund den Schleim aus den Luftwegen anssog, könnten vielleicht als experimentelle Beweise betrachtet werden.

Die Sicherheit der Fiitterungstuberculose, welche znerst von Gerlach experimentell hervorgernfen wurde, und durch eine grosse Reihe von Experimentatoren ${ }^{1}$ ") zuletzt namentlich durch Ko $\mathrm{ch}^{11}$ ) Bestätigung fand, ist gegenwärtig ebenfalls allgemein anerkannt. Als besonders charakteristisch sind die Versuche von Aufrech ${ }^{12}$ ) zu betrachten, welcher kur'z nach dem Wurfe weibliche Kaninchen inficirte, deren Junge nachher durch die Lactation tuberculös wurden.

Der Neugeborene kanm anf dem Wege der natürlichen oder künstlichen Ernährung inficirt werdeı. Nach Analogie des oben angeführten Aufrecht'schen Thierexperinentes zn schliessen, wïrde nun auch eine tuberculöse Mntter ihren Sängling inficiren kömuen, wofïr wir allerdings zur Zeit noch keine mathematisch genauen $\mathrm{Be}$ weise haben. Es lässt sich das nur aus der täglichen Frfahrung als sehr wahrscheinlich und vielleicht sicher bezeichnen. Epstein ${ }^{13}$ ) hält die Milch schwindsüchtiger Mütter für besonders gefälırlich, nach seinen Beobachtmingen kam es nie zur Entwickelung von Tuberculose be Kindern tulerculöser Abstammung, wenn sie an die Brıst gesunde Ammen gelegrt wurden. Herterich ${ }^{14}$ ) erwähnt einen Fall, wo eine durch den schwindsüchtigen Ehegatten inficirte Fran durch die Lactation die Tuberculose auf ihre beiden Kinder übertrug. Die von mir oben angeführte Beobachtıng, wo 2 Ehefrauen, nach einander

1) Bockendahl, Ergebnisse d. Schwindsuchtsstatistik d. Vereins. Mit theilnngen d. Vereins Schleswig-Holstein. Aerzte. VI. 127. VII. 101 Separatabdruck. 12.13

2) Uffelmann, Jahresbericht, Supplem.-Bd. d. Deutschen Vierteljahrschr f. off. Gcsundheitspfl. 1884. 30 .

3) Ewald, Aetiologie, Diagnose, Prognose: Behandlung d. Tuberculose. Berl. klinische Wochenschrift 1884,44

${ }^{4}$ ) Tappeiner, Virchow's Archiv Bd. 74, 393. Bd. 82. 353

5) Johne, a. a. 0.29

6) Veraguth, a. a. ()

7 Zeitschrift d. Wiener Aerute 1883,2

8) Lajoue, Recherches exper. sur la contag. de la tuberculose, Thèse Nancy. 1883.

9 Berlin. klin. Woclienschr 1878,37

10) Johne, a. a. 0.31

13) Koch, Aetiologie d. Tubercul. a. a. 0

12) Medic. Centralbl. 1882. $289 \mathrm{f}$

13) Epstein, Prager Vierteliahresschr. N. F. II 1879.103

14) Aerztl. Intelligenzbl. 1883. 26. durch denselben Ehemanı angesteckt, walırscheinlich ebenfalls durch das Säugungsgeschäft die Krankheit anf ihre Kinder ïbertrugen, könnte hier anch mitgerechnet werden. Ich bin iiberzeugt, dass bei genauen Nachforschungen sich viele solche Beispiele anfüliren lassen dürften.

Bezüglich der Infection durch kiinstliche Ernährung des Neugeborenen ist zunächst zll erwähnen, wie neuerdings von $\mathrm{May}^{2}$ ) über die Infectiosität der Milch perlsüchtiger Kühe im Bollinger'schen Institnte seln wichtige Versuche angestellt worden sind, ans denen zweifellos hervorgeht, dass die ungekocht genossene Milch von Kühen, welche an allgemeiner Tuberculose leiden, wieder Tuberculose hervorzurufen im Stande ist. Beispiele für die Erzeugung von Schwindsucht beim Menschen durch den Genuss von Milch perlsüchtiger Kühe sind von Klebs ${ }^{2}$ ) erwähnt, und $Z$ ii $\mathrm{rn}^{3}$ ) macht anf die Häufigkeit der Tuberculose beim Menschen in Gegenden, wo viel perlsüchtiges Vieh existirt, anfmerksam. Cohnheim ${ }^{4}$ ) meint, dass die so oft bei Kindern auftretenden Darmphthisen anf die künstliche Ernährnııg durch Milch von tıberculösen Kühen zuriickzufühıren seien. De $\mathrm{mme} \mathrm{e}^{5}$ ) conlstatirte in 4 Fällen von Darmtuberculose durch den Nachweis volı Bacillen den ursächlichen Zusammenhang mit dem Genuss roher Milch einer perlsiichtigen Kuh, hält aber diese Verbreitungsweise der Tuberculose wegen der geringen Anzahl einschlägiger Beobachtungen für eine seltene; Zippelius ${ }^{6}$ ) constatirte anf Grund einer 5jälırigen Statistik ans seinem Amtsbezirke, dass die Sterblichkeit der Kinder unter 2 Jalıren in den Orten am grössten war, wo nach Ausweis der Fleischschan die meisten tuberculösen Kühe sich befanden.

Lis scheint, als hätte man diesem ätiologischen Momente bisher noch zı wenig Beachtung geschenkt, die Zahl einschllägiger Beobachtungen dïrfte sich bei genaueren Nachforschungen woll sicherlich vergrösseln. Ich glaube sogar annehmen zu dürfen, dass solche lnfectionsfälle viel häufiger sind, denn eine nicht unerhebliche Anzahl von Atrophieen und chronischen Darmkatarrhen im frïhesten Kindesalter sind vermuthlich auch anf Tuberculose zurïckznfïliren. lch bin zu dieser Annahme durch die Thatsache verleitet worden, dass die Tubercnlosensterblichkeit bei Kindern in den ersten Lebensjahren in Bezng anf die monatlichen Elevationen dem Typus der Ern̈̈lırnıgskrankheiten folgt $n$ nd mit diesen die Spitze der Conrve im Sommer anfweist, während die allgemeine Mortalitätscurve der Schwindsucht und die der höheren Altersklassen zwei Curvenberge im Früljalır und Winter hat. ${ }^{7}$ ) Die Kindersterblichkeit in den ersten Jahren wird im Allgemeinen im Sommer von den Todesfällen in Folge von Ernälnrungs-Krankheiten beherrscht, und ans der Aehnlichkeit der genannten beiden Curven habe ich auch eine gewisse Verwandtschaft in ätiologischer Beziehnng nicht fï̈r unwalnrscheinlich gehalten. Ich liabe deshalb angenommen, dass in den ersten Lebensjahren die intestinalen Formen der Tuberculose recht hänfig sind, während in den anderen Altersklassen melır die pulmonalen überwiegen. Dabei liegt es mun nahe, an gewisse mutritive Vorgänge zu denken, und bei der Häufigkeit der Perlsucht nnter den Küllen einerseits, andererseits hei der immer aligemeiner werdenden limährung des Kindes durch Kulmilch ist die Vermuthnng gewiss berechtigt und naheliegend, dass einc Infection durch den Genuss roher Milch perlsüchtiger Kühe doch in der 'That hänfiger sein möchte a]s man lisher glaubte. Die Annahme von Klebs ${ }^{\mathrm{y}}$ ), dass der Darn eine Hanptinvasionsstelle für das Tuberkelgift sei, dürfte beziiglich des kindlichen Alters dadurch an Wahrscheinlichkeit gewinnen, wenn anch Biedert ${ }^{9}$ ) anf Grund einer eingehenden Analyse des hierhergehörigen Materiales sich dahin änssert, dass die Gefahr der Infection des Menschen durch den Darm keine selır dringende sei.

Es ist noch zu erwähnen. dass muter Umständen auch läsionen der Hant als Invasionsstellen für die Bacillen dienen können. Demme ${ }^{10}$ ) behauptet dies von den läufigen chronischen Eczemen und impetiginösell Ansschlägen. Von 823 Fällen tuberculöser Knochen- und Gelenkerkrankungen waren $437=53$ Proc. vorher mit derartigen sogen. scrofulösen Alisschlägen behaftet gewesen, und neuerdings hat derselbe bei 2 Fällen von Eczem im Bläscheninhalte Bacillen gefunden. Kleine Läsionen der Haut, Wunden können den Bacillen den Fintritt in den Körper gestatten und so Veranlassung zu örtlichen und auch allgemeinen Tuberculosen werden, freilich liegen keine reichlichen Beob-

1) May, im Archiv f. Ilygiene, 1883. 1. Heft 121 .

Klebs, Virchow's Archiv Bd. 49. 291

3) Zürn, zoopatholog. u. zoophysiolog. Untersuchungen 1871. 7 .

4) Cohnheim, a. a.0. 25

D emme, XX. Jahresber. d. Jenner'schen Kinderspitals 1882. 48.

6) Zippelius (Wochenschrift f. Thierheilk. u. Viehz: XX, 225.)

7) Wahl, a. a. O. 118

5) Klebs, Verhandl. d. II. Congr. f. inner. Med. II. 53.

9) Biedert. Die Tuberculose d. Darmes u. des lymphat. Apparates, Jahırb. f. Kinderhlk. N. F. p. 171.

10) Demme, a. a. 0. 48 , u. XXI Bericht 28 
achtungen vor und $\mathrm{K}_{0} \mathrm{enig}^{1}$ ) meint, dass es so gut wie gar keine Beweise dafür gäbe, dass bei einem gesunden Menschen das tuberculöse Virus von einer Wunde aus eindringe. Die einzige dafür sprechende von ihm gemachte Beobachtung der Entstehnng localer und von da aus verbreiteter Tuberculose in einer Tibiawunde bei einem rachitischen Kinde bezeichnet er selbst als nicht ganz einwandsfrei. Bollinge ''s $^{2}$ ) Versuche über cutane Impfungen von Tuberculose ergaben ein negatives, subcutane ein positives Resultat.

(Fortsetzung folgt.) 